\title{
Tunneling through the Quantum Dot Coupled between Normal and Superconducting Leads
}

\author{
A. Donabidowicz, T. Domański and K.I. Wysokiński \\ Institute of Physics, M. Curie-Skłodowska University \\ Radziszewskiego 10, 20-031 Lublin, Poland
}

\begin{abstract}
We study the charge tunneling via the quantum dot coupled to normal and superconducting leads, where the superconducting electrode has either an isotropic or anisotropic (of $d$-wave symmetry) energy gap. We use the single impurity Anderson model and apply the nonequilibrium Green function formalism to determine the differential tunneling conductance. The influence of the proximity effect between the quantum dot and superconductor on the transport properties of the system manifests itself in the Andreev conductance.
\end{abstract}

PACS numbers: 73.23.-b, 74.20.Fg, 74.45.+c

\section{Introduction}

The ability to fabricate and operate the solid state quantum devices of nanometer size such as quantum dots, quantum wires, and single molecules opens exciting perspectives for the future nanotechnology. Their unique transport properties can be used for constructing various nanodevices. Recently there has been observed a growing interest in studying the single electron transistor (SET) consisting of the conducting source, superconducting drain, and the quantum dot located in-between [1-7].

In the structures with both normal electrodes the discrete energy spectrum of the dot leads to oscillations of the tunneling conductance. If one electrode is replaced by a superconductor there arise some qualitatively new effects. They are related with activation of anomalous transport channels such as the Andreev current. This phenomenon is sensitive to the symmetry of the order parameter and we will illustrate it here by comparing the results for isotropic and anisotropic superconductors.

\section{Theoretical description}

We assume that the quantum dot (QD) coupled to the normal and superconducting leads can be described by the single impurity Anderson Hamiltonian 


$$
\begin{aligned}
H= & \sum_{\sigma} \epsilon_{d} d_{\sigma}^{+} d_{\sigma}+U n_{d \uparrow} n_{d \downarrow}+\sum_{\boldsymbol{k}, \beta, \sigma}\left(V_{\boldsymbol{k} \beta} c_{\boldsymbol{k} \beta \sigma} d_{\sigma}^{+}+V_{\boldsymbol{k} \beta}^{*} c_{\boldsymbol{k} \beta \sigma}^{+} d_{\sigma}\right) \\
& +\sum_{\boldsymbol{k}, \beta, \sigma} \xi_{\boldsymbol{k} \beta} c_{\boldsymbol{k} \beta \sigma}^{+} c_{\boldsymbol{k} \beta \sigma}-\sum_{\boldsymbol{k}}\left(\Delta_{\boldsymbol{k}} c_{\boldsymbol{k} S \uparrow}^{+} c_{-\boldsymbol{k} S \downarrow}^{+}+\text {h.c. }\right) .
\end{aligned}
$$

Operators $c_{\boldsymbol{k} \beta \sigma}\left(c_{\boldsymbol{k} \beta \sigma}^{+}\right)$annihilate (create) electrons in the normal $(\beta=\mathrm{N})$ or the superconducting $(\beta=\mathrm{S})$ electrodes with the corresponding energies $\xi_{\boldsymbol{k} \beta \sigma}=$ $\epsilon_{\boldsymbol{k} \sigma}-\mu_{\beta}$ measured from the chemical potentials which in a presence of the external voltage $V$ are shifted by $\mu_{\mathrm{N}}-\mu_{\mathrm{S}}=e V$. Operators $d_{\sigma}, d_{\sigma}^{+}$refer to the localized electrons of the dot. The QD electrons are characterized by the single energy level $\epsilon_{d}$ and a potential $U$ of the on-dot Coulomb repulsion. $V_{\boldsymbol{k} \beta}$ describes the hybridization between the localized and itinerant electrons.

To determine the charge current $J(V)=-e\left\langle\dot{N}_{\mathrm{L}}\right\rangle$ we have to resort to the nonequilibrium Keldysh Green function formalism. This problem is exactly solvable only if one omits the Coulomb interactions $U$. Otherwise some further approximate treatments have to be used in order to handle the correlation effects. The Dyson equation $G_{d}^{\mathrm{r}}(\omega)^{-1}=g_{d}^{\mathrm{r}}(\omega)^{-1}-\Sigma_{0}(\omega)-\Sigma_{\mathrm{I}}(\omega)$ involves the matrix self-energies where the contribution $\Sigma_{0}(\omega)$ corresponds to the noninteracting case $(U=0)$ and the part $\Sigma_{\mathrm{I}}(\omega)$ is due to correlation effects. We focus here on the simple (but non-trivial) case of the uncorrelated QD when $\Sigma_{\mathrm{I}}(\omega)=0$. A more general case $U \neq 0$ will be discussed separately.

\section{Results}

Assuming that the energy gap of superconducting lead is isotropic over the Fermi surface $\Delta_{k}=\Delta$ we investigate the energy spectrum of QD and conductance of the tunneling system. In the left hand side part of Fig. 1 we show the density
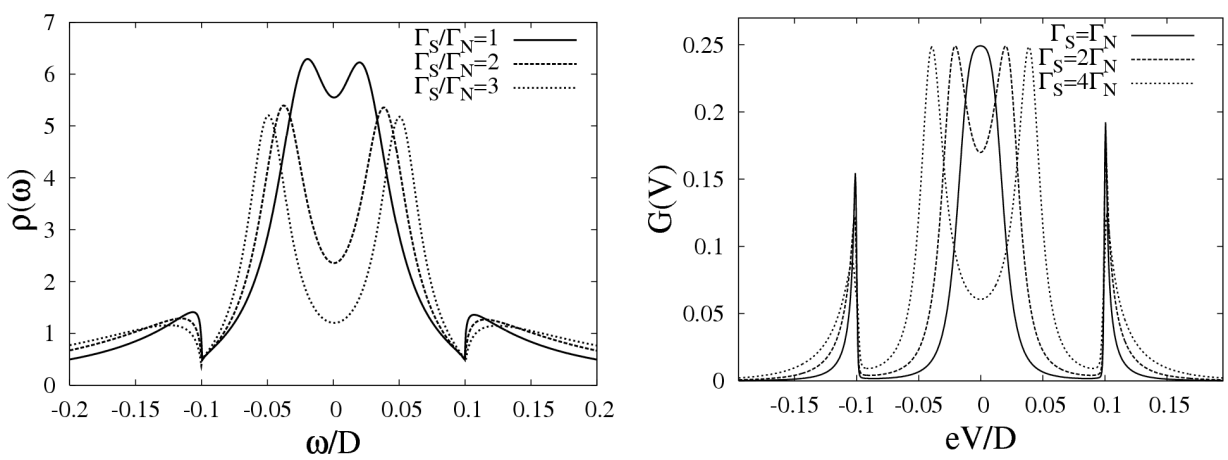

Fig. 1. Spectral function $\rho(\omega)$ of the uncorrelated $(U=0)$ quantum dot coupled to a normal lead and $s$-wave superconductor with $\Delta=0.1 D$ and QD level $\varepsilon_{d}=0$. We considered $\left|\varepsilon_{\boldsymbol{k}}\right| \leq D$ and used a finite band width $D$ as a unit for the energies. 
of QD. We notice that the energy level $\varepsilon_{d}$, which is located in the center of superconducting gap, splits into two peaks due to the particle-hole mixing induced by the proximity effect. It has been noted by Tanaka et al. [7] that the broadening of peaks is given by $\Gamma_{\mathrm{N}}$ while their mutual distance is controlled by $\Gamma_{\mathrm{S}}$ (here $\Gamma_{\beta}$ is the coupling between the quantum dot and $\beta$ lead, which we assume to be constant).

The structure of the QD spectral function $\rho(\omega)$ has a direct impact on the differential conductance $G(V)=\mathrm{d} J / \mathrm{d} V$. In the right hand side part of Fig. 1 we show the low temperature conductance versus the bias $V$. Let us emphasize that the in-gap conductance (for $|e V| \leq \Delta$ ) arises from the Andreev current.

In superconductors with $d$-wave symmetry of the order parameter the energy gap varies over the Fermi surface $\Delta_{\boldsymbol{k}}=\Delta\left(\cos k_{x} a-\cos k_{y} a\right)$ vanishing along the nodal directions $k_{x}= \pm k_{y}$ hence the corresponding quasiparticle density of states is gapless. Because of symmetry reasons [8] the on-dot Green function $G_{d}^{\mathrm{r}}(\omega)$ becomes diagonal (no proximity effect). This has a substantial influence on the spectral function $\rho(\omega)$ (shown in Fig. 2) characterized by a single peak structure.
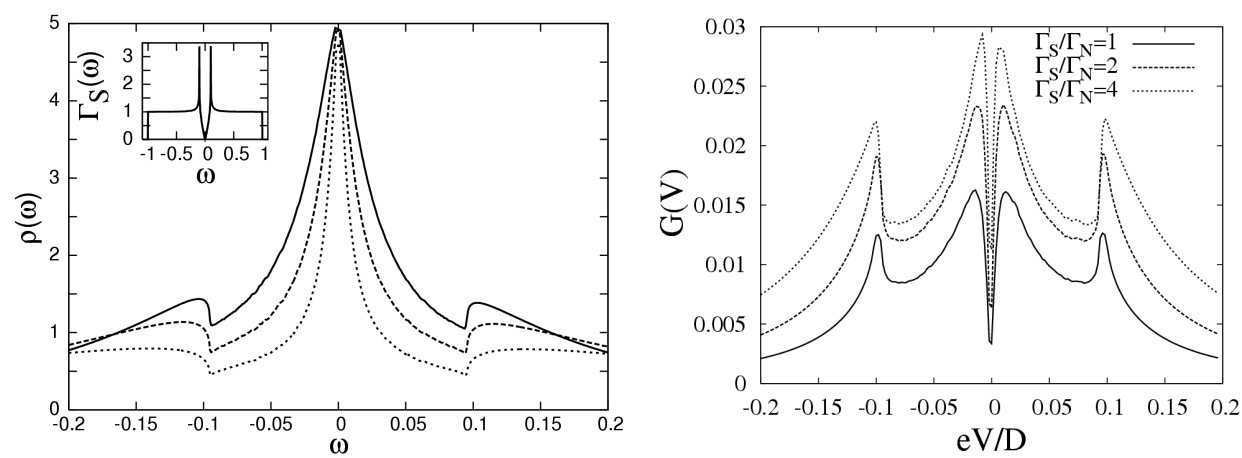

Fig. 2. The same results as in Fig. 1 but for the $d$-wave superconductor. Inset shows the energy dependence of the QD coupling to $d$-wave superconductor.

This peak can be further modified due to correlation effects [9] but even for $U=0$ its width is strongly affected by the energy dependence of $\Gamma_{\mathrm{S}}(\omega)$ (see the inset in Fig. 2 on the left). The absence of proximity effect suppresses all the anomalous tunneling channels [10] (including the Andreev current) therefore the differential conductance qualitatively differs in comparison to isotropic superconductor. In Fig. 3 we illustrate $G(V)$ for the QD coupled to the isotropic and $d$-wave superconductors for a set of energy levels $\varepsilon_{d}$.

In summary, we studied the tunneling through the quantum dot located between the normal and superconducting leads with either the isotropic or anisotropic ( $d$-wave) order parameter. For the isotropic superconductor we find that the quantum dot absorbs the off-diagonal ordering (the proximity effect) and this strongly affects the charge transport at low bias $|e V| \leq \Delta$. Because of the particle-hole mixing there appear two well-separated in-gap maxima which show 

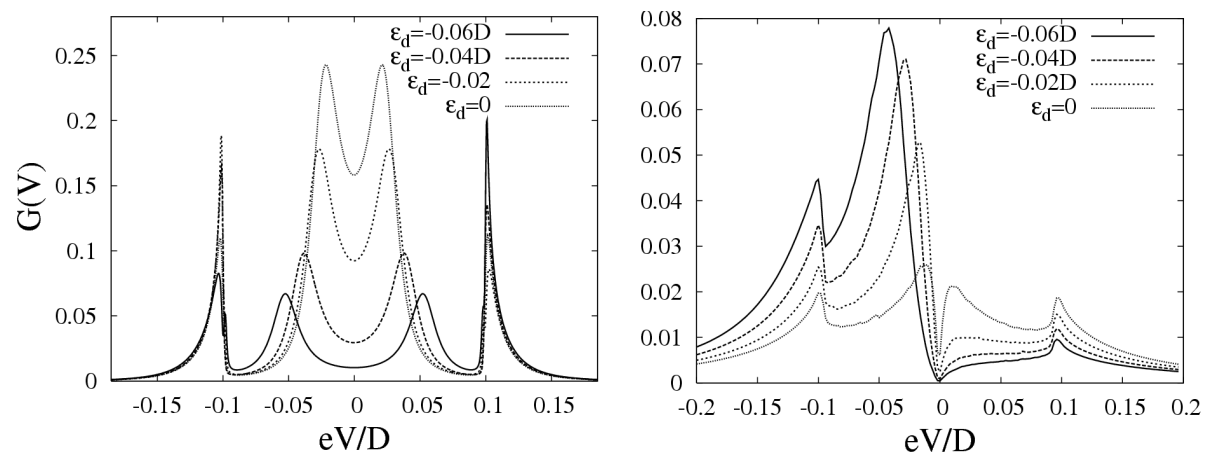

Fig. 3. Voltage dependence of the differential conductance $G(V)$ for the quantum dot with $U=0$ and $\Gamma_{\mathrm{N}}=\Gamma_{\mathrm{S}}$. The figure illustrates the influence of QD energy level $\varepsilon_{d}$ for the $s$-wave (left part) and $d$-wave (right part) superconductor.

up in the Andreev conductance. For the $d$-wave superconducting lead the proximity effect is absent. In consequence, the tunneling occurs only through the usual normal channel and the differential conductance is suppressed at small voltage $V$.

\section{Acknowledgments}

This work was supported by Ministry of Science and Higher Education.

\section{References}

[1] J.E. Mooij, Y.V. Nazarov, Nature Phys. 2, 169 (2006).

[2] R. Fazio, R. Raimondi, Phys. Rev. Lett. 80, 2913 (1998); Phys. Rev. Lett. 82, 4950 (1998).

[3] Q.-F. Sun, J. Wang, T.-H. Lin, Phys. Rev. B 59, 3831 (1999).

[4] A.A. Clerk, A. Ambegaokar, S. Hirschfield, Phys. Rev. B 61, 3555 (2000).

[5] J.C. Cuevas, A. Levi Yeyati, A. Martin-Rodero, Phys. Rev. B 63, 094515 (2001).

[6] M. Krawiec, K.I. Wysokiński, Supercond. Sci. Technol. 17, 103 (2002).

[7] Y. Tanaka, N. Kawakami, A. Oguri, cond-mat/0701570.

[8] L.S. Borkowski, P.J. Hirschfeld, Phys. Rev. B 46, 9274 (1992).

[9] M. Vojta, R. Bulla, Phys. Rev. B 65, 014511 (2002); A. Polkonikov, S. Sachdev, M. Vojta, Phys. Rev. Lett. 86, 296 (2001).

[10] G.E. Blonder, M. Tinkham, T.M. Klapwijk, Phys. Rev. B 25, 4515 (1982). 